\title{
Direct Approach to Compute a Class of Reaction-Diffusion Equation by a Finite Element Method
}

\author{
Sadia Akter Lima ${ }^{1}$, Md. Kamrujjaman ${ }^{2,3, *}$, Md. Shafiqul Islam ${ }^{1}$ \\ ${ }^{1}$ Department of Applied Mathematics, University of Dhaka, Dhaka 1000, Bangladesh. \\ ${ }^{2}$ Department of Mathematics, University of Dhaka, Dhaka 1000, Bangladesh. \\ ${ }^{3}$ Department of Mathematics and Statistics, University of Calgary, Calgary, AB, Canada.
}

How to cite this paper: Sadia Akter Lima, Md. Kamrujjaman, Md. Shafiqul Islam. (2020) Direct Approach to Compute a Class of Reaction-Diffusion Equation by a Finite Element Method. Journal of Applied Mathematics and Computation, 4(2), 26-33. DOI:10.26855/jamc.2020.06.003

Received: April 17, 2020

Accepted: May 10, 2020

Published: May 18, 2020

"Corresponding author: Md. Kamrujjaman, Department of Mathematics, University of Dhaka, Dhaka 1000, Bangladesh; Department of Mathematics and Statistics, University of Calgary, Calgary, AB, Canada.

Email: kamrujjaman@du.ac.bd

\begin{abstract}
In this study, we consider the robust and well known numerical method such as Finite Element Method (FEM) to find the numerical approximation of nonlinear parabolic partial differential equations (PDEs). The key objective of this research paper is to study the numerical solution of the famous FitzHugh-Nagumo equation and Fisher's equation with regular and irregular geometrical shapes. The numerical scheme used here is a finite element method (FEM) in a simple and convenient way. We mainly focus to find out the accuracy and acceptance of this method by applying small time step size. To convey the efficiency of this method for solving the nonlinear equation, the results are portrayed both graphically and in tabular form which demonstrate the efficiency of this algorithm. The method can be applied for solving any nonlinear parabolic partial differential equations (PDEs).
\end{abstract}

\section{Keywords}

Finite Element Method, FitzHugh-Nagumo, nonlinear PDE, Galerkin FEM, Numerical solution

\section{Motivation}

In mathematics, partial differential equations (PDEs) hold unfamiliar multivariable functions, their partial derivatives and environ rates of change with respect to continuous variables. Basically, our real life problems can be modeled by PDEs with applications to engineering, physics, chemistry, ecology, biology, and other important fields of science. Different forms of PDEs are mainly

- linear or nonlinear;

- homogeneous or nonhomogeneous;

- elliptic, hyperbolic, or parabolic.

As like others, PDEs also contain some specific characteristics which are enable to provide information how smooth the required solution is, also helps us to know what are the impacts of initial and boundary conditions. These equations are used to explain an extensive variety of phenomena such as electrostatics, quantum mechanics, electrodynamics, fluid dynamics, elasticity, gravitation, sound, heat and diffusion. Parabolic equation is one of the affiliate of PDE and has many important applications of parabolic equations, from a parabolic antenna or parabolic microphone to automobile headlight reflectors to the design of ballistic missiles. Most of the practical problems which are represented by any mathematical model formed by nonlinear PDEs. To create some solvable PDEs, many other assumptions have been made. 
These nonlinear PDEs which are capable to show our real life problems sometimes become difficult to solve analytically. For finding the solutions of those nonlinear PDEs many researchers have invented some methods due to their potential uses and for better and more efficient solutions of those PDEs they gave more devotation [1].

In 2004, Wazwaz, A. M. and Gorguis, A. [2] developed a work to get the exact solution to Fisher's equation and to a nonlinear diffusion equation of the Fisher type by employing Adomian decomposition method. Ahmed, A. and Kamrujjaman, M. [3] considered the nonlinear parabolic Fisher's equations for travelling wave solutions. They focused on to discuss the analytic solution in the spatial pattern of travelling wave solutions. Introducing various techniques, the modified Burger's and Burger's-Fisher equation were solved in $[4,5,6]$.

Hodgkin and Huxley introduced FitzHugh-Nagumo (F-N) equation

$$
\frac{\partial U}{\partial t}=\frac{\partial^{2} U}{\partial x^{2}}+U(U-\lambda)(1-U) ; 0 \leq \lambda \leq 1
$$

because of it is importance in various fields of science and the branches are:

- logistic population growth;

- branching Brownian motion process and circuit theory;

- the transmission of nerve impulses;

- the area of population genetics.

Hariharan, G. and Kannan, K. [7] developed an accurate and efficient Haar wavelet method for solving FitzHugh Nagumo equation and this scheme can be used to a wide class of nonlinear reaction-diffusion equations. There are only a very few events where it is possible to exist analytic solutions of such reaction-diffusion equations. Due to this reason, many researchers have felt the emergency to construct accurate and efficient numerical methods and among those methods first of all the Finite Difference Method (FDM) raises potential outcomes in our working field. The next one is the computational cost to achieve higher accuracy. To overcome this tenacious situation, during some years Finite Element Method (FEM) plays the most important role for solving problems of engineering and mathematical physics with complicated geometries, loadings and material properties. Islam, Ahmed, and Hossain introduced finite element method to solve the IVP and BVP using Taylor Series, integration technique and using Bernoulli polynomials $[8,9]$. In Yingjun, J. and Jingtang, M. [10] proposed to develop high-order methods for solving time-fractional partial differential equations based on high-order Finite Element Method for space and finite difference method for time the method was developed. Modified Fitzhugh-Nagumo (F-N) equation [7] is a nonlinear diffusion-reaction equation. Last two decades, this nonlinear parabolic PDE has been found many important applications in fields as Brownian motion process, circuit theory, transmission of nerve impulses, population genetics, neuron dynamics and neurophysiology, flame propagation, autocatalytic chemical reaction, nuclear reactor theory etc. $[11,12]$. Due to its importance in recent years, few works have been completed in order to obtain the numerical solution of this equation [13]- [16].

The key objective of our study is to analysis the numerical approximation of FitzHugh-Nagumo equation (1.1) and Fisher's equation as presented in Section 3; reaction-diffusion equations of the form of PDEs by using FEM. For better understanding, we derive the general formulation of FEM. To investigate the efficiency of this method, the results are exhibited both graphically and numerically and declares the acceptance of it for solving any nonlinear parabolic partial differential equation (PDE).

In the following section, we discuss about the formulation of FEM for a class of second order nonlinear parabolic PDEs.

\section{A FEM for Diffusion-Reaction Equations}

To find approximate solution of different problems of our real life and in different fields of science, a competent numerical approach is very important to introduce. Considering this, let's introduce the most effective and efficient numerical method that is Finite Element Method. In this section, we formulate the Finite Element Method for the numerical solution of Diffusion-Reaction equation which is an important mathematical model in wide range of applications both in natural sciences and engineering. These applications include the transport of air, adsorption of pollutants in soil, diffusion of neutrons, modelling of biological systems, oil reservoir flow transport and reaction of chemical species etc., are the applications of this equations and among those applications, in the governing equation used unknown variables represent the non-negative values of physical quantities such as pollutants, population, concentration of chemical compounds. To derive formulation for FEM let consider the following diffusion-reaction (DR) model with dirichlet boundary conditions:

$$
\begin{array}{crl}
\frac{\partial U}{\partial t}=d \frac{\partial^{2} U}{\partial x^{2}}+f(U(x, t)) ; & (x, t) \in[\alpha, \beta] \times[0, T] \\
U(0, x) & =U_{0}(x) ; & x \in[\alpha, \beta] \\
U(t, \alpha)=U_{\alpha}(t) ; & t \in[0, T]
\end{array}
$$




$$
U(t, \beta)=U_{\beta}(t) ; \quad t \in[0, T]
$$

In this proposed equation $\frac{\partial^{2} U}{\partial x^{2}}$ is the diffusion term, $d=$ the diffusion coefficient, $f(U(x, t))$ is the reaction term. Here, $U_{\alpha}(t)$ and $U_{\beta}(t)$ represent the functions of time, $t$ and $U_{0}(x)$ represents the function of spatial variable, $x$. To derive this formulation, let consider the trial solution

$$
\widetilde{U}(x, t)=\sum_{j=1}^{n} a_{j}(t) \Psi_{j}(x)
$$

where the parameter $a_{j}$ be the functions of $t$. For this kind of problems, we are interested to use two linear shape functions

$$
L_{1}(\xi)=\frac{1-\xi}{2}, L_{2}(\xi)=\frac{1+\xi}{2} ; \xi \in[-1,1]
$$

Instead of using quadratic and other shape functions, linear shape functions are preferable to use because it is easier and give better accuracy [12]. Hence the weighted residual equation for this DR equation for a particular element $[e]$ is

Which yields

$$
\begin{aligned}
& \int_{[e]}\left[\frac{\partial \widetilde{U}}{\partial t}-d \frac{\partial^{2} \widetilde{U}}{\partial x^{2}}-f(\widetilde{U}(x, t))\right] \Psi_{i}(x) d x=0 \\
\Rightarrow & \int_{[e]}\left[\frac{\partial \widetilde{U}}{\partial t} \Psi_{i}(x)-d \frac{\partial^{2} \widetilde{U}}{\partial x^{2}} \Psi_{i}(x)-f(\widetilde{U}(x, t)) \Psi_{i}(x)\right] d x=0
\end{aligned}
$$

$$
\int_{[e]} \frac{\partial \widetilde{U}}{\partial t} \Psi_{i}(x) d x+d \int_{[e]} \frac{d \psi_{i}}{d x} \frac{\partial \widetilde{U}}{\partial x} d x-\int_{[e]} f(\widetilde{U}) \psi_{i}(x) d x=d\left[\frac{\partial \widetilde{U}}{\partial x} \psi_{i}(x)\right]_{[e]}
$$

At this stage, after using equation (2.2) and simplification the usual matrix form can be written as:

Where

$$
C_{i, j} \frac{d a_{j}(t)}{d t}+K_{i, j} a_{j}(t)=F_{i}
$$

and

$$
C_{i, j}=\int_{[e]}\left(\sum_{j=1}^{n} \Psi_{j}\right) \Psi_{i}(x) d x, \quad F_{i}=d\left[\frac{\partial \widetilde{U}}{\partial x} \psi_{i}(x)\right]_{[e]}
$$

We explore the right side of (2.7) as

$$
K_{i, j}=D_{i, j}+S_{i, j}
$$

$$
D_{i, j}=d \int_{[e]} \frac{d \psi_{i}}{d x} \frac{d \psi_{j}}{d x} d x ; S_{i, j}=-\int_{[e]} f\left(\sum_{j=1}^{n} \psi_{j}\right) \Psi_{i} d x
$$

Usually, $C_{i, j}$ is the capacity or heat capacity integral and known as capacity matrix. In equation (2.7) $D_{i, j}$ is obtained from diffusion term, $S_{i, j}$ from source term. After assembling we get the following convenient matrix form:

$$
C \dot{U}+K U=\mathrm{F}
$$

Equation (2.8) represents a system of first order ordinary differential equations with one independent variable $t$ and we can discretize $\dot{U}\left(=\dot{a}_{j}\right)$ as

$$
\dot{U}=\frac{U_{j+1-U_{j}}}{\Delta t}
$$

and we have to use a particular value of $\Delta t$. After introducing equation (2.9) and completing a simple calculation equation (2.8) provides us

$$
C U_{j+1}+(K \Delta t-C) U_{j}=F \Delta t
$$

The nodal values $U_{j+1}$ can be obtained by using row-column operation and for first approximation one may choose the initial values obtained from initial condition.

\section{Numerical Results and Discussion}

In this section, for better understanding, we apply this FEM for finding approximate solution of FitzHugh-Nagumo equation and Fisher's equation. After a sedulous observation, eventually we will show a good matching between approximate and exact solutions with a negligible error that tends to zero for small time steps.

Example 1. In this section, we proposed the scheme as discussed in the previous section and we consider the simulation of the modified Fitzhugh-Nagumo equation [7] to validate the general formulation of FEM. Here, the 
boundary conditions of this F-N equation have boundless smoothness for sustaining the accuracy of the proposed scheme. To originate the numerical solutions of this initial boundary value problems, we have used the MATLAB programming platform. Assuming $\lambda=1$, the modified Fitzhugh-Nagumo equation is:

with initial condition

$$
\frac{\partial U}{\partial t}=\frac{\partial^{2} U}{\partial x^{2}}+U(U-1)(1-U) ;(x, t) \in[1,5] \times[0, T]
$$

and the boundary conditions

$$
U(0, x)=\frac{1}{2}\left[1-\operatorname{coth}\left(\frac{-x}{2 \sqrt{2}}\right)\right] ; x \in[1,5]
$$

$$
\begin{aligned}
& U(t, 1)=\frac{1}{2}\left[1-\operatorname{coth}\left(\frac{-1}{2 \sqrt{2}}+\frac{t}{4}\right)\right], t>0 \\
& U(t, 5)=\frac{1}{2}\left[1-\operatorname{coth}\left(\frac{-5}{2 \sqrt{2}}+\frac{t}{4}\right)\right], t>0
\end{aligned}
$$

The exact solution of the equation (3.1) is

$$
U(x, t)=\frac{1}{2}\left[1-\operatorname{coth}\left(\frac{-x}{2 \sqrt{2}}+\frac{t}{4}\right)\right]
$$

Approximate solution has been computed by using the derived formulation of our proposed scheme in order to validate

\begin{tabular}{|c|c|c|c|c|c|c|c|c|c|}
\hline \multirow{2}{*}{$\mathrm{x}$} & \multicolumn{3}{|c|}{$\mathrm{h}=0.0001$} & \multicolumn{3}{|c|}{$\mathrm{h}=0.001$} & \multicolumn{3}{|c|}{$\mathrm{h}=0.005$} \\
\hline & FEM & Exact & Error & FEM & Exact & Error & FEM & Exact & Error \\
\hline 1.0 & 5.2852 & 5.2849 & $3.0 \times 10^{-04}$ & 5.2891 & 5.2857 & $3.4 \times 10^{-03}$ & 5.3064 & 5.2891 & $1.7 \times 10^{-02}$ \\
\hline 1.4 & 4.6589 & 4.6409 & $1.8 \times 10^{-02}$ & 4.6747 & 4.6412 & $3.4 \times 10^{-02}$ & 4.7445 & 4.6427 & $1.0 \times 10^{-01}$ \\
\hline 1.8 & 4.3581 & 4.3404 & $1.8 \times 10^{-02}$ & 4.3685 & 4.3406 & $2.8 \times 10^{-02}$ & 4.4148 & 4.3413 & $7.4 \times 10^{-02}$ \\
\hline 2.2 & 4.2009 & 4.1865 & $1.4 \times 10^{-02}$ & 4.2118 & 4.1866 & $2.5 \times 10^{-02}$ & 4.2601 & 4.1870 & $7.3 \times 10^{-02}$ \\
\hline 2.6 & 4.1146 & 4.1038 & $1.0 \times 10^{-02}$ & 4.1249 & 4.1039 & $2.1 \times 10^{-02}$ & 4.1706 & 4.1041 & $6.7 \times 10^{-02}$ \\
\hline 3.0 & 4.0662 & 4.0583 & $7.9 \times 10^{-03}$ & 4.0764 & 4.0583 & $1.8 \times 10^{-02}$ & 4.1216 & 4.0585 & $6.3 \times 10^{-02}$ \\
\hline 3.4 & 4.0386 & 4.0329 & $5.7 \times 10^{-03}$ & 4.0487 & 4.0329 & $1.6 \times 10^{-02}$ & 4.0933 & 4.0330 & $6.0 \times 10^{-02}$ \\
\hline 3.8 & 4.0228 & 4.0186 & $4.2 \times 10^{-03}$ & 4.0328 & 4.0186 & $1.4 \times 10^{-02}$ & 4.0772 & 4.0187 & $5.9 \times 10^{-02}$ \\
\hline 4.2 & 4.0137 & 4.0106 & $3.2 \times 10^{-03}$ & 4.0237 & 4.0106 & $1.3 \times 10^{-02}$ & 4.0681 & 4.0106 & $5.8 \times 10^{-02}$ \\
\hline 4.6 & 4.0084 & 4.0060 & $2.4 \times 10^{-03}$ & 4.0182 & 4.0060 & $1.2 \times 10^{-02}$ & 4.0620 & 4.0060 & $5.6 \times 10^{-02}$ \\
\hline 5.0 & 4.0053 & 4.0034 & $1.9 \times 10^{-03}$ & 4.0156 & 4.0034 & $1.2 \times 10^{-02}$ & 4.0613 & 4.0034 & $5.7 \times 10^{-02}$ \\
\hline
\end{tabular}
this scheme with exact solution (3.4) of the modified Fitzhugh-Nagumo equation. In that case, the finite number of elements is taken as $n=10$ and two linear shape functions as defined in equation (2.3). We completed the numerical computation and that numerical values at different time steps and spatial distributions, which are tabulated below.

Table 1. FEM solutions and exact solution of modified Fitzhugh-Nagumo equation (3.1).

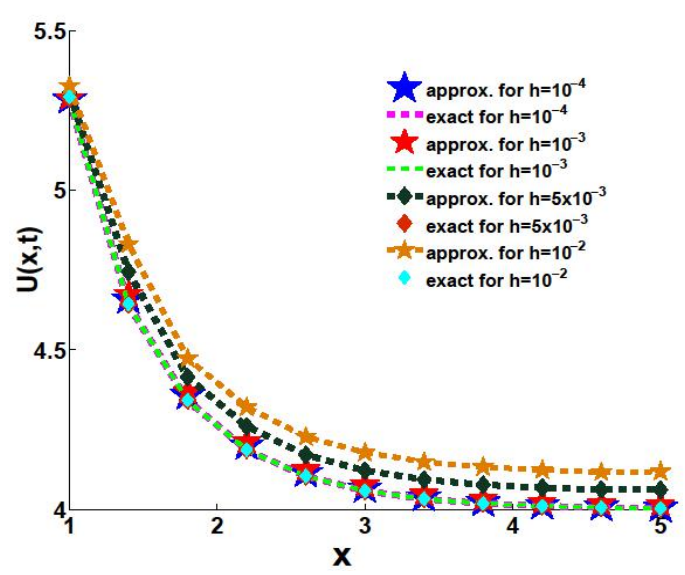

Figure 1. The plot of approximate and exact results of equation (3.1). 

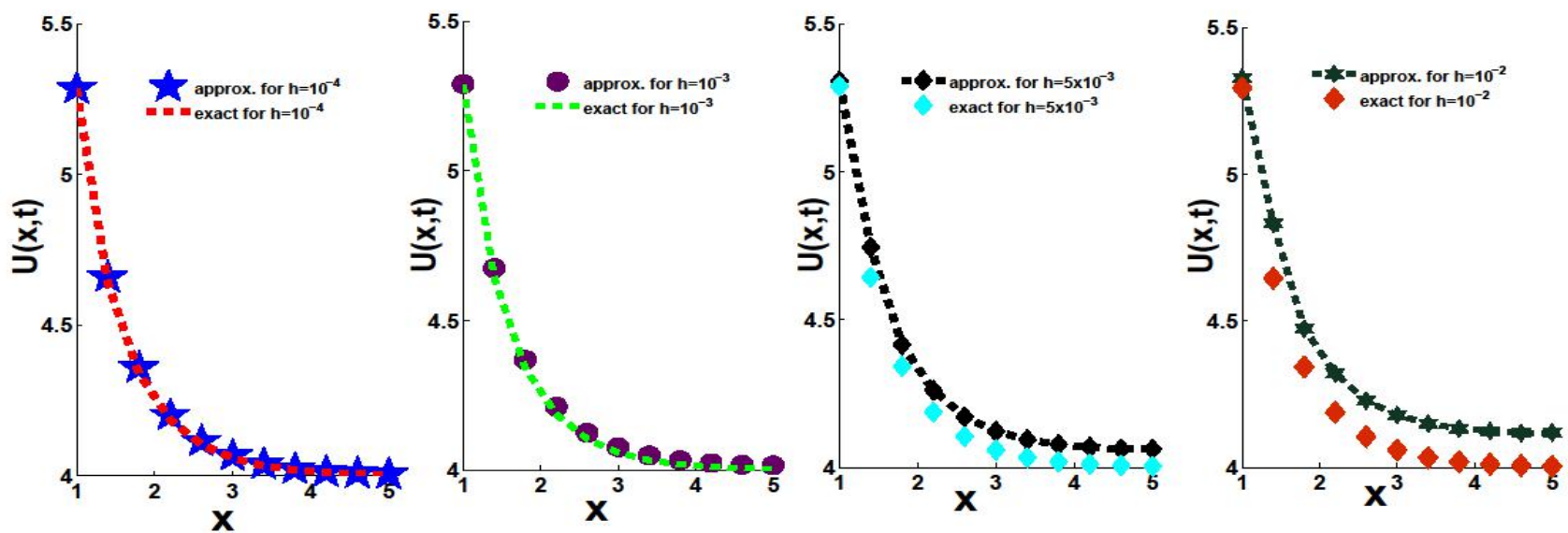

Figure 2. Graphical relationship between the approximate and exact solution of equation (3.1).

From Table 1, it is seen that our proposed method gives a good accuracy over the domain for different time circles. A good similarity can be obtained between the characteristics of approximate solution with the exact solution.

Figures 1 and 2 are the required results obtained from equation (3.1) by using the established algorithm of our introduced method, FEM for various time steps. Figures 1 and 2 exhibit the correspondence between two graphs of approximate and exact results where the error term is quite omit-able. They also ensure the accuracy and acceptance of our proposed scheme, although inherently the error is feasible for comparatively larger time steps.

For better understanding, let us set up the three dimensional surface plot, Figure 3 of the numerical solution of modified Fitzhugh-Nagumo equation (3.1). From this type of comparison, it is hard to distinguish. That is why we also include the absolute error map over time $t$ and space $x$. Eventually, it makes sense clearly that this method is more applicable to solve such nonlinear parabolic PDEs without any complexity and maintains a perfect consent between this FEM solution and exact solution (3.4).
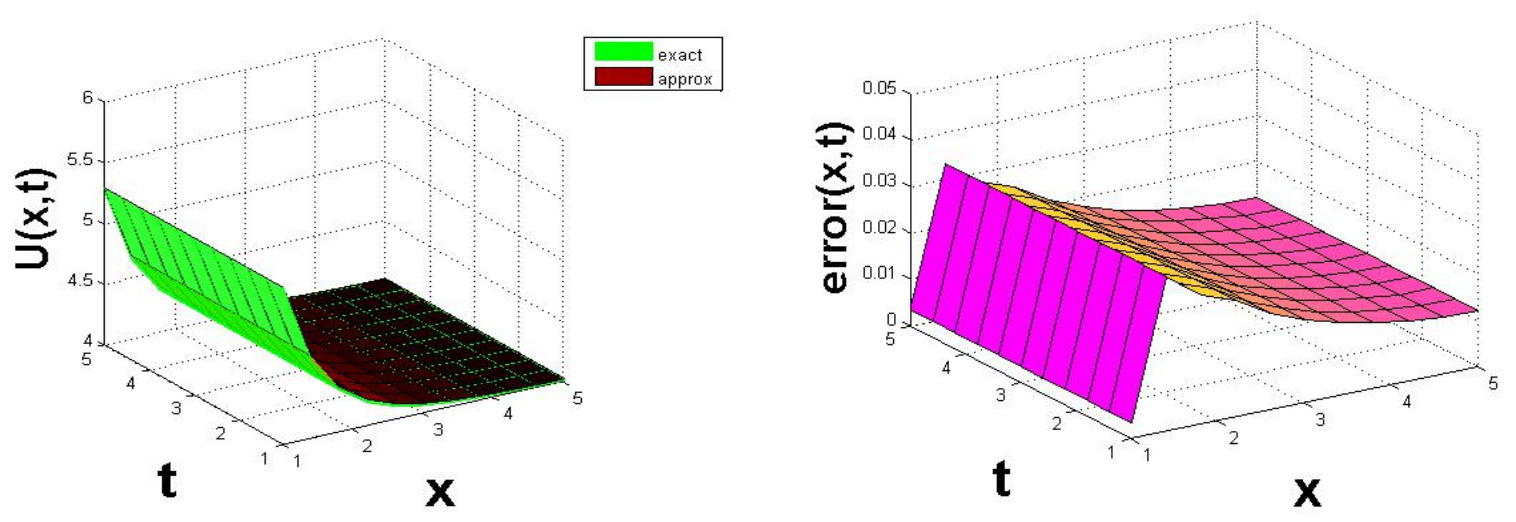

Figure 3. Numerical solutions of modified Fitzhugh-Nagumo equation (3.1) and absolute error map for $h=\Delta t=0.001$.

Example 2: Fisher's equation is a reaction-diffusion equation with the inhomogeneous term $f(U(x, t))=\mu U(1-U)$ and consider growth rate $\mu=6$, diffusion coefficient $d=1$, then from the DR model (2.1) we obtain the well-known Fisher's equation [3]

$$
\frac{\partial U}{\partial t}=\frac{\partial^{2} U}{\partial x^{2}}+6 U(1-U) ;(x, t) \in[0,1] \times[0, T]
$$

The boundary condition and initial condition are given by the equations (3.6) and (3.7) respectively.

$$
\begin{aligned}
& U(t, 0)=\left(1+e^{-5 t}\right)^{-2}, t>0 \\
& U(t, 1)=\left(1+e^{1-5 t}\right)^{-2}, t>0 \\
& U(0, x)=\left(1+e^{x}\right)^{-2}, x \in[0, T]
\end{aligned}
$$

The exact solution to this problem is $U(t, x)=\left(1+e^{x-5 t}\right)^{-2}$. 
To find the numerical solution of equation (3.5), explicit and implicit finite difference method was introduced in [3]. We apply the technique of FEM on this time, dependent partial differential equation i.e., Fisher's equation (3.5), according to the procedure discussed in the previous section. We will try to represent the required approximate solution both numerically in tabular form and graphically in diagram. To ensure the accuracy and acceptance of this method, we will take an attempt to make a comparison between the approximate numerical solution and exact solution. To find numerical computation, let consider finite number of elements, $n=10$, linear shape functions and several time steps $\mathrm{h}=\Delta \mathrm{t}$. Particularly, the convenient matrix form provides us

Where

$$
C U_{j+1}+(K \Delta t-C) U_{j}=F \Delta t
$$

$$
\begin{gathered}
K_{i, j}=D_{i, j}+S_{i, j}, C_{i, j}=\int_{[e]} \Psi_{i}(x)\left(\sum_{j=1}^{n} \Psi_{j}(x)\right) d x \\
D_{i, j}=\int_{[e]} \frac{d \Psi_{i}}{d x} \frac{d \Psi_{j}}{d x} d x, \mathrm{~F}(\mathrm{t})=\left[\frac{\partial \widetilde{U}}{\partial x} \psi_{i}(x)\right]_{[e]} \\
S_{i, j}=6 \int_{[e]} \psi_{i}(x)\left(\sum_{j=1}^{n} \psi_{j}(x)\right)\left(a_{1}(t) \psi_{1}(x)+a_{2}(t) \Psi_{2}(x)\right) d x-6 \int_{[e]} \psi_{i}(x)\left(\sum_{j=1}^{n} \psi_{j}(x)\right) d x
\end{gathered}
$$

Table 2. Corelative study between approximate and exact solution for different values of $h=\Delta t$ of Fisher's equation (3.5).

\begin{tabular}{cccccccccc}
\hline & & $\mathrm{h}=0.001$ & & & $\mathrm{~h}=0.01$ & & & $\mathrm{~h}=0.1$ \\
$\mathrm{x}$ & $\mathrm{FEM}$ & Exact & Error & FEM & Exact & Error & FEM & Exact & Error \\
\hline 0.0 & 0.2508 & 0.2513 & $5.0 \times 10^{-04}$ & 0.2579 & 0.2627 & $4.7 \times 10^{-03}$ & 0.3299 & 0.2756 & $5.4 \times 10^{-02}$ \\
0.1 & 0.2268 & 0.2267 & $1.0 \times 10^{-04}$ & 0.2370 & 0.2377 & $7.0 \times 10^{-04}$ & 0.3408 & 0.2500 & $9.1 \times 10^{-02}$ \\
0.2 & 0.2036 & 0.2038 & $2.0 \times 10^{-04}$ & 0.2117 & 0.2140 & $2.3 \times 10^{-03}$ & 0.2947 & 0.2256 & $6.9 \times 10^{-02}$ \\
0.3 & 0.1819 & 0.1821 & $2.0 \times 10^{-04}$ & 0.1895 & 0.1917 & $2.2 \times 10^{-03}$ & 0.2665 & 0.2026 & $6.4 \times 10^{-02}$ \\
0.4 & 0.1618 & 0.1620 & $2.0 \times 10^{-04}$ & 0.1685 & 0.1709 & $2.4 \times 10^{-03}$ & 0.2362 & 0.1811 & $5.5 \times 10^{-02}$ \\
0.5 & 0.1432 & 0.1434 & $2.0 \times 10^{-04}$ & 0.1491 & 0.1516 & $2.5 \times 10^{-03}$ & 0.2093 & 0.1611 & $4.8 \times 10^{-02}$ \\
0.6 & 0.1261 & 0.1264 & $3.0 \times 10^{-04}$ & 0.1314 & 0.1339 & $2.5 \times 10^{-03}$ & 0.1845 & 0.1425 & $4.2 \times 10^{-02}$ \\
0.7 & 0.1106 & 0.1108 & $2.0 \times 10^{-04}$ & 0.1152 & 0.1176 & $2.5 \times 10^{-03}$ & 0.1615 & 0.1256 & $3.6 \times 10^{-02}$ \\
0.8 & 0.0966 & 0.0968 & $2.0 \times 10^{-04}$ & 0.1007 & 0.1029 & $2.2 \times 10^{-03}$ & 0.1424 & 0.1101 & $3.2 \times 10^{-02}$ \\
0.9 & 0.0839 & 0.0841 & $2.0 \times 10^{-04}$ & 0.0870 & 0.0897 & $2.6 \times 10^{-03}$ & 0.1188 & 0.0961 & $2.3 \times 10^{-02}$ \\
1.0 & 0.0728 & 0.0729 & $1.0 \times 10^{-04}$ & 0.0772 & 0.0778 & $6.0 \times 10^{-03}$ & 0.1217 & 0.0836 & $3.8 \times 10^{-02}$ \\
\hline
\end{tabular}

It has been clearly understood from Table 2, our mentioned FEM ensures a good accuracy for different time steps $h=\Delta t$. Also Figure 4 exhibits the approximate and the analytical solutions for various time steps $h=\Delta t$. This diagram helps us to belief that the numerical solution is as harmonic as the exact solution.

From Figure 5, we can observe that the 3D graphical representation of the correlative study between the exact and a finite element approximation. Since the FEM solution is almost similar to the exact solution, so from this diagram, it is not straight forward and easy to find out the difference between them. To overcome this difficult situation, the approximate solution is exhibited by a transparent graph. In order to understand clearly, we also affix an error map over the independent variable time $t$ and space variable $x$ which displays the accuracy of this method by showing a good compliance with the exact solution. At last but not least, we can say that the error tends to zero and declares the acceptance of this FEM for solving parabolic PDEs. 


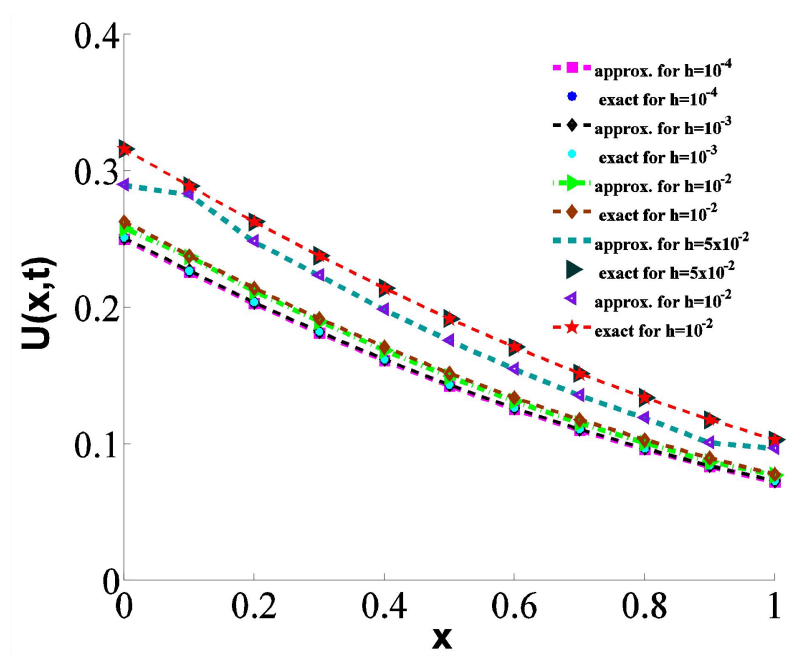

Figure 4. Presentation of FEM solution $\widetilde{U}(x, t)$ and the exact solution $U(x, t)$ of equation (3.5) for different time steps $h=\Delta t$.
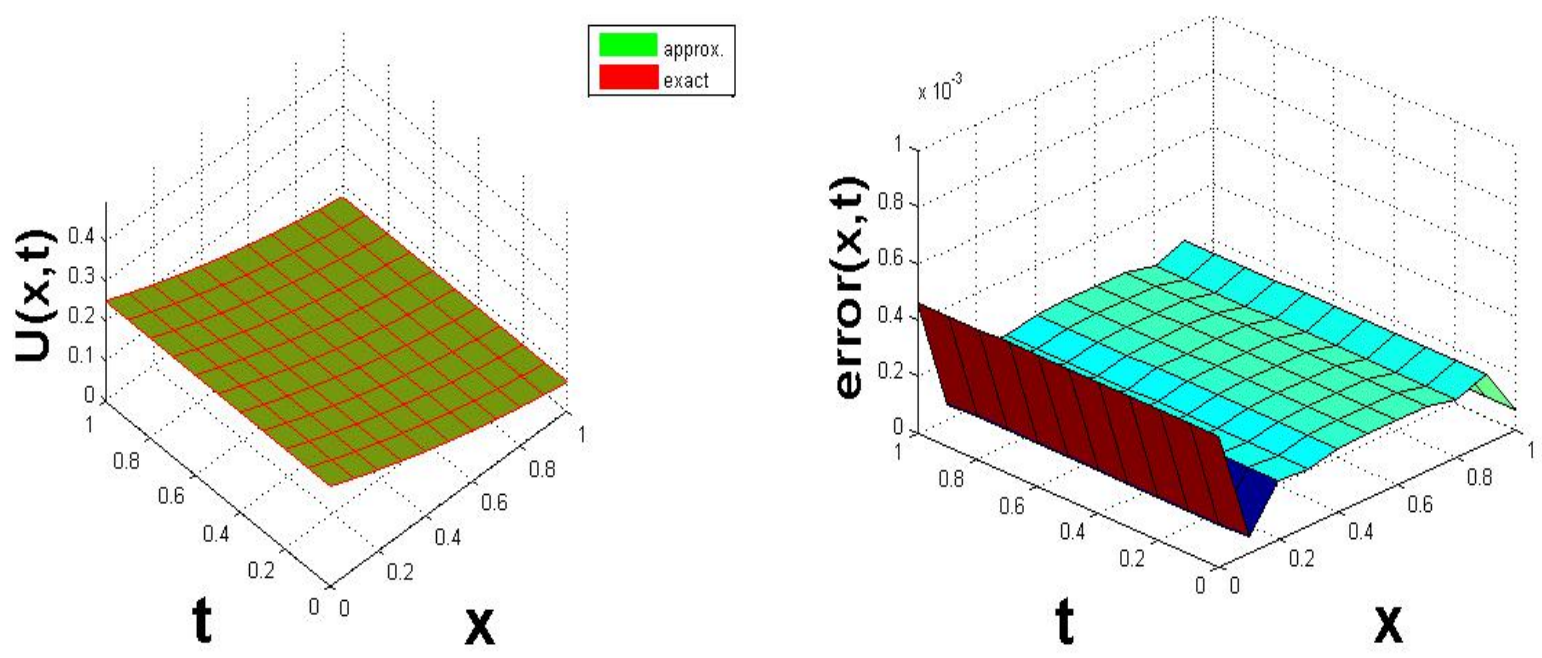

Figure 5. Correlative study of equation (3.5) and absolute error map for $h=\Delta t=0.001$.

\section{Conclusion}

In this paper, we derived the complete formulation of Finite Element Method (FEM) for diffusion-reaction (DR) model as well as nonlinear parabolic PDEs. The FEM has been displayed and formulated to solve a large class of nonlinear problems; particularly we considered the modified FitzHugh-Nagumo equation and Fisher's equation with the approximate solution which convergent are more rapidly to exact solution. To obtain the approximate solution of these two equations, the method has been successfully employed. The obtained numerical results are quite satisfactory for a wide range of time steps as well as space steps and the absolute error map provides very less amount of error that can be neglected. Data structured table and graphical maps of approximate and exact solution provide a cool agreement for wide range of time and space steps. Afterwards, it is clear from both numerical and graphical presentation that the characteristics of all solutions are as harmonic as the exact and the solutions are convergent and accurate of higher order that also show the rapid convergence of this FEM than any other available numerical methods. At last but not least, we may conclude by declaring the efficiency, accuracy and acceptance of this proposed robust FEM because of its simplicity, low cost, time saving properties, easy formulation and good consent carrying capability with the exact solution obtained from well-published literature. 


\section{References}

[1] Lewis, P. E. and Ward, J. P. (1991). The Finite Element Method (Principles and Applications). Wokingham: Addison-Wesley, March 1991.

[2] Wazwaz, A. M. and Gorguis, A. (2004). An analytic study of Fisher's equation by using Adomian decomposition method. Applied Mathematics and Computation, 154(3), 609-620.

[3] Ahmed, A. and Kamrujjaman, M. (2019). Analytic Travelling Wave Solutions and Numerical Analysis of Fisher's Equation via Explicit-Implicit FDM. Asian Journal of Advanced Research and Reports, 3(3), 1-13.

[4] Sungnul, S., Jitsom, B. and Punpocha, M. (2018). Numerical Solution of the Modified Burger's Equation using FTCS Implicit Scheme. IAENG International Journal of Applied Mathematics.

[5] Kocacoban, D., Koc, A. B., Kurnaz, A., and Keskin, Y. (2011). A Better Approximation to the Solution of Burger-Fisher Equation. World Congress on Engineering, 1.

[6] Behzadi, S. S. (2011). Numerical Solution for Solving Burger's-Fisher Equation by Using Iterative Methods. Mathematical and Computational Applications, 16(2), 443-455.

[7] Hariharan, G. and Kannan, K. (2010). Haar wavelet method for solving FitzHugh Nagumo equation. Int. J. Comput. Math. Sci, 4(7), 909-913.

[8] Islam, M. S., Ahmed, M., and Hossain, M. A. (2010). Numerical Solutions of IVP using Finite Element Method with Taylor Series, GANIT: Journal of Bangladesh Mathematical Society, 30, 51-58.

[9] Islam, M. S. and Shirin, A. (2011). Numerical solutions of a class of second order boundary value problems on using Bernoulli polynomials. Applied Mathematics, 2, 1059-1067.

[10] Yingjun, J. and Jingtang, M. (2011). High-order Finite Element Methods For Time Fractional Partial Differential Equations. Journal of Computational and Applied Mathematics, 235, 3285-3290.

[11] Zhangxin, C. (2005). Finite element methods and their applications. Springer Science and Business Media.

[12] Reddy, J. N. (2014). An Introduction to Nonlinear Finite Element Analysis, OUP, Oxford.

[13] Chen, Z., Gumel, A. B. and Mickens, R. E. (2003). Nonstandard discretizations of the generalized Nagumo reaction-diffusion equation, Numerical Methods for Partial Differential Equations: An International Journal, 19(3), 362-379.

[14] Feng, H. and Lin, R. (2015). A finite difference method for the FitzHugh-Nagumo equations: Dynamics of Continuous, Discrete and Impulsive Systems, Series B: Applications and Algorithms, 22, 401-402.

[15] Van Gorde, R. A. (2012). Gaussian waves in the FitzHugh-Nagumo equation demonstrate one role of the auxiliary function $\mathrm{h}(\mathrm{x}, \mathrm{t})$ in the homotopy analysis method, Communications in Nonlinear Science and Numerical Simulation, 17(3), 1233-1240.

[16] Teodoro, M. F. (2012). Numerical approximation of a nonlinear delay-advance functional differential equation by a finite element method. AIP Conference Proceedings, American Institute of Physics, 1479, 806-809. 